\title{
Surgical outcomes of hepatocellular carcinoma with biliary tumor thrombus: a systematic review
}

\author{
Wenhui Qiao ${ }^{1+}$, Feng $\mathrm{Yu}^{2+}$, Lupeng $\mathrm{Wu}^{3}$, Bin $\mathrm{Li}^{3}$ and Yanming Zhou ${ }^{3 *}$
}

\begin{abstract}
Background: Hepatocellular carcinoma (HCC) with biliary tumor thrombus (BTT) is rare and its impact on postoperative prognosis remains controversial. The aim of this study was to evaluate the published evidence concerning the outcome of surgical resection of HCC with BTT.

Methods: Eligible studies were identified by searching PubMed and reviewed systematically. Comparisons of the clinicopathologic features and surgical outcomes for HCC patients with or without BTT were analyzed using meta-analytical techniques.

Results: Twenty retrospective studies containing 598 patients that met the selection criteria were included for review. The perioperative mortality was $2.1 \%$ (range, 0-10\%), and the median 5-year overall survival (OS) was $24 \%$ (range, 0-48 \%) with a recurrence rate of $63.9 \%$ (range, 42-91\%). Pooled analysis of 13 comparative studies showed that HCC patients with BTT had a higher incidence of vascular invasion (odds ratio [OR]: 4.70, $95 \% \mathrm{Cl}$ : 2.90-7.60; $P<0.001$ ), a higher frequency of poor differentiation (OR: 2.07, $95 \% \mathrm{Cl}: 1.23-3.49 ; P=0.006$ ), and a shorter 5 -year OS rate (OR: $0.31,95 \% \mathrm{Cl}: 0.21-0.64 ; P<0.001)$ than those without BTT.

Conclusions: Although HCC with BTT has more aggressive biological characteristics and is an indicator of poor prognosis, surgical resection can still provide long-term survival for some patients.
\end{abstract}

Keywords: Hepatocellular carcinoma, Biliary tumor thrombus, Resection, Prognosis

\section{Background}

Hepatocellular carcinoma $(\mathrm{HCC})$ is the fifth most common neoplasm in the world and is the third leading cause of cancer-related death worldwide, with more than 500000 new cases diagnosed each year [1]. Surgical resection remains the mainstay of curative treatment for this disease. Portal vein thrombus is a frequent event in $\mathrm{HCC}$ and has important impact on patient survival after surgical resection [2], while biliary tumor thrombus (BTT) is relatively rare with a reported incidence of $0.53-12.9 \%$ in autopsy and surgical specimens [3-9]. In this regard, few reports are available in the literature

\footnotetext{
* Correspondence: zhouymsxy@sina.cn

${ }^{\dagger}$ Equal contributors

${ }^{3}$ Department of Hepato-Biliary-Pancreato-Vascular Surgery, First affiliated Hospital of Xiamen University, Xiamen, China

Full list of author information is available at the end of the article
}

addressing the role of surgical resection for this special clinical entity. In addition, the prognostic impact of BTT is controversial $[5,6,9]$. The current study assesses the published literature on surgical resection for $\mathrm{HCC}$ with BTT and compares the clinicopathologic features and long-term postoperative outcomes between HCC patients with BTT and those without BTT.

\section{Methods}

Systematic search strategy

A comprehensive systematic review of all published literature from 1966 to September 2015 was undertaken using PubMed database. The following Medical Subject Headings terms were used: "hepatocellular carcinoma," "biliary tumor thrombus," "bile duct tumor thrombus," and "bile duct thrombus." Reference lists of all retrieved articles were manually searched to identify further potentially relevant articles. This study was performed

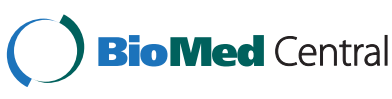

(c) 2016 Qiao et al. Open Access This article is distributed under the terms of the Creative Commons Attribution 4.0 International License (http://creativecommons.org/licenses/by/4.0/), which permits unrestricted use, distribution, and reproduction in any medium, provided you give appropriate credit to the original author(s) and the source, provide a link to the Creative Commons license, and indicate if changes were made. The Creative Commons Public Domain Dedication waiver (http://creativecommons.org/publicdomain/zero/1.0/) applies to the data made available in this article, unless otherwise stated. 
according to Preferred Reporting Items for Systematic Reviews and Meta-analyses (PRISMA) guideline guidelines [10].

\section{Inclusion and exclusion criteria}

English language studies with a sample size of at least 10 patients that reported long-term survival data following surgical resection for $\mathrm{HCC}$ with BTT were included. Animal studies, letters, reviews, abstracts, editorials, expert opinions, duplicates, studies with fewer than 10 patients, studies involving patients who were treated with nonsurgical management or liver transplantation were excluded.

\section{Data extraction and quality assessment}

Information on study design, first author, country or region, year of publication, study population characteristics, and outcomes of interest were independently extracted by two authors (Yanming Zhou and Feng Yu). Discrepancies between the two reviewers were resolved by discussion and consensus. Study methodology quality was categorized according to the Evidence-based Medicine Levels of Evidence [11].

Macroscopic BTT was defined when it was present in the common hepatic duct or the first to second branches of the bile duct and microscopic BTT was defined when it was present in the third order or more peripheral branches of the bile duct [12].

The primary outcome measures were 1-, 3- and 5-year overall survival (OS) following surgical resection.

\section{Statistical analysis}

Data extracted for BTT group were reported as total and percentage for categorical variables and as median values and range for continuous variables, unless otherwise stated. The results of comparative studies of patients with BTT and without BTT were pooled by the use of Review Manager.software, version 5.1 (The Cochrane Collaboration, Software Update, Oxford). Dichotomous variables were expressed as odds ratio (OR) with a $95 \%$ confidence interval $(95 \% \mathrm{CI})$, and continuous variables were expressed as the weighted mean difference (WMD) with a $95 \%$ CI. $\chi^{2}$ test and $\mathrm{I}^{2}$ were used to assess heterogeneity between studies. The random-effect model was used if there was significant heterogeneity $(P<0.1)$; otherwise, the fixed-effect model was used. Publication bias was evaluated via funnel plot. Statistical significance was set at $P<0.05$.

\section{Results}

\section{Systematic review}

Among 154 potentially relevant papers identified by the initial search, 20 finally met the inclusion criteria in this review and are summarised in Table 1 [3-9, 12-26]. All studies were retrospectively designed, originated from Asia (Japan, $n=8[5,6,12,13,15,20,21,25]$; Mainland
China, $n=7[7,8,14,16,17,19,26]$; Korea, $n=2$ [18, 22]; Taiwan, $n=1$ [9]; India, $n=1$ [23]; Hong Kong, $n=1$ [24]) and classified as level-4 evidence. The sample size of each study varied from 13 to 73 patients. Thirteen studies utilised patients without BTT as a control group for comparison [5, 6, 9, 13, 15-17, 19-24].

The included papers described 598 patients who underwent surgical resection for HCC with BTT, including $526(88 \%)$ patients who had macroscopic BTT and 72 (12\%) patients who had microscopic BTT. Most $(80.2 \%, 441 / 550)$ patients were men. The median or mean age ranged from 47.1 to 60 (median $=54.2$ ) years . Hepatitis B surface antigen (HBsAg) and hepatitis C virus antibody (Anti-HCV) were positive in $60.7 \%(324 / 533)$ and $14.9 \%(50 / 335)$ of patients, respectively. Mean or median tumor size ranged from 3.8 to $6.4($ median $=5.4) \mathrm{cm}$. Cirrhosis, tumor multiplicity, vascular invasion, poor differentiation, intrahepatic metastasis, and tumor capsule absence, occurred in $60.4 \%$ (229/379), $36.8 \%$ (127/345), $67.1 \%$ (306/456), $47.9 \%$ (188/392), $31.6 \%$ (92/291), and $66.7 \%(140 / 210)$ of patients, respectively.

Table 2 shows the operative intervention and postoperative outcomes. Among 573 patients with available information, hepatectomy with or without tumor thrombectomy was carried out in 423 (73.8 \%), followed by hepatectomy combined bile duct resection in $144(25.1 \%)$, thrombectomy only in $4(0.7 \%)$, and thrombectomy with hepatic artery ligation and cannulation in $2(0.35 \%)$. There were 13 perioperative deaths $(2.1 \%)$ (range, $0-10 \%)$. The median overall survival was 26.1 months (range, 11.4-47 months). The median 1-, 3- and 5-year OS rates were $72 \%$ (range, $38-93 \%$ ), $39 \%$ (range, 11-77 \%) and 24 \% (range, 0-48 \%), respectively. Disease recurrence developed in $64.8 \%$ (310/ 478) patients.

\section{Meta-analysis}

A total of 13 comparative studies including 334 patients with BTT and 6361 without BTT were included for analysis $[5,6,9,13,15-17,19-24]$. Table 3 presents a summary of outcomes. Table 3 presents a summary of the outcomes. There was no significant difference in clinicopathological characteristics between the two groups in terms of sex, age, hepatitis viral status (hepatitis B or C virus), presence of cirrhosis, tumor size and multiplicity, tumor capsule absence, and intrahepatic metastasis, while vascular invasion (OR: 4.70, 95 \% CI: 2.90-7.60; $P<0.001$ ) and poor differentiation (OR: $2.07,95 \% \mathrm{CI}$ : $1.23-3.49 ; P=0.006)$ were more frequently observed in BTT group than those in non-BTT group.

The 1-, 3- and 5-year OS rates in patients with BTT were significantly lower than those in patients without BTT (OR: 0.48, 95 \% CI: 0.31-0.73; $P<0.001$; OR: 0.45, 95 \% CI: 0.29-0.680; $P<0.001$; OR: 0.31, 95 \% CI: $0.21-$ $0.64 ; P<0.001$, respectively) (Figs. 1, 2 and 3 ). 
Table 1 Clinical background of included studies

\begin{tabular}{|c|c|c|c|c|c|c|c|c|c|c|c|c|c|}
\hline First author (Year) & No. & $M / F$ & Age (years) ${ }^{a}$ & BTT type Ma/Mi & HBsAg n (\%) & Anti-HCV n (\%) & Cirrhosis n (\%) & $\mathrm{TS}(\mathrm{cm})^{\mathrm{a}}$ & MT n (\%) & VI n (\%) & PD n (\%) & IM n (\%) & TCA n (\%) \\
\hline Satoh (2000) [5] & 17 & $15 / 2$ & 58.2 & $17 / 0$ & $5(29.4)$ & - & - & - & - & $11(64.8)$ & $5(29.4)$ & - & - \\
\hline Shiomi (2001) [6] & 17 & $15 / 2$ & 58.8 & $17 / 0$ & $7(46.7)$ & $3(17.6)$ & $6(35 \%)$ & 6.1 & $12 / 16(75)$ & $13(76)$ & $0(0)$ & $5(29)$ & - \\
\hline Peng (2004) [7] & $15^{b}$ & $10 / 5$ & 49 & $15 / 0$ & $13(86.7)$ & - & - & 5.1 & $2(13.3)$ & $5(33.3)$ & - & - & - \\
\hline Qin (2004) [8] & 34 & $28 / 6$ & 48.5 & $34 / 0$ & $34(100)$ & - & $32(94.1)$ & 6.4 & - & - & - & - & $34(100)$ \\
\hline Yeh (2004) [9] & 17 & $14 / 3$ & 52.3 & $17 / 0$ & - & - & - & 3.8 & - & $12 / 16(75)$ & $3 / 11(27.2)$ & 5/16 (83.3) & 2/11 (18.2) \\
\hline Esaki (2005) [12] & 38 & $32 / 6$ & 62 & $19 / 19$ & $8(21.1)$ & - & $10(26.3)$ & 6.2 & $19(50)$ & $27(71)$ & $11(28.9)$ & $19(50)$ & - \\
\hline Ikenaga (2009) [13] & 15 & $12 / 3$ & 66 & $10 / 5$ & $4(27.7)$ & $7(46.7)$ & - & 5.0 & $6(40)$ & 12/14 (85.7) & - & $10(66.7)$ & $2(13.3)$ \\
\hline Luo (2009) [14] & $48^{c}$ & - & - & $48 / 0$ & - & - & - & 6.2 & - & - & - & - & - \\
\hline Noda (2011) [15] & 22 & $21 / 1$ & 58 & $22 / 0$ & $15(68.1)$ & $5(22.7)$ & $6(27.2)$ & $>5, n=9$ & $20(90.9)$ & $13(59)$ & $18(81.8)$ & - & - \\
\hline Shao (2011) [16] & 27 & $24 / 3$ & 47.1 & $24 / 3$ & $26(96.7)$ & $0(0)$ & $18(66.7)$ & $>5, n=10$ & - & $17(62.9)$ & $26(96.3)$ & $5(18.5)$ & $21(77.7)$ \\
\hline Yu (2011) [17] & $20^{d}$ & $17 / 3$ & 50.6 & $20 / 0$ & $16(80)$ & - & $14(70)$ & 4.5 & - & $12(60)$ & $13(65)$ & $4(20)$ & $15(75)$ \\
\hline Moon (2013) [18] & 73 & $52 / 21$ & 54.2 & $73 / 0$ & $59(80.8)$ & $2(2.8)$ & $62(84.9)$ & 5.8 & $11(15)$ & $53(72.6)$ & $54(74)$ & $25(34.2)$ & - \\
\hline Meng (2014) [19] & 35 & $24 / 11$ & 51.3 & $28 / 7$ & $26(74.3)$ & $0(0)$ & $25(65.8)$ & $>5, n=24$ & $15(42.8)$ & $10(25.8)$ & $10(25.8)$ & - & $24(68.6)$ \\
\hline Oba (2014) [20] & 13 & $12 / 1$ & 61 & $13 / 0$ & $4(30.7)$ & $5(38.5)$ & $3(23)$ & 4.4 & $2(15.4)$ & $12(92.3)$ & - & - & - \\
\hline Kasai (2015) [21] & 44 & $35 / 9$ & 64 & $44 / 0$ & $8(18.2)$ & $21(52.5)$ & $14(31.8)$ & 5.8 & $16(36.4)$ & $31(70.4)$ & $18(40.9)$ & - & - \\
\hline Kim (2015) [22] & 31 & $21 / 10$ & 53 & $0 / 31$ & $26(83.9)$ & $1(3.2)$ & - & 4.8 & - & $28(90.3)$ & $1(3.2)$ & $8(25.8)$ & $7(22.5)$ \\
\hline Rammohan (2015) [23] & 39 & $28 / 11$ & 52.1 & $39 / 0$ & $7(17.9)$ & $2(5.1)$ & Excluded & 5.6 & - & - & - & - & - \\
\hline Wong (2015) [24] & 37 & $29 / 8$ & 57 & $37 / 0$ & $30(81.1)$ & - & - & 6 & $15(29.7)$ & $25(67.6)$ & $11(29.7)$ & - & 35 (94.6) \\
\hline Yamamoto (2015) [25] & 19 & $19 / 0$ & 67 & $19 / 0$ & $7(36.8)$ & $4(21)$ & $6(31.5)$ & 4.3 & - & - & - & - & - \\
\hline Zeng (2015) [26] & 37 & $33 / 4$ & 50 & $30 / 7$ & $29(78.4)$ & - & $33(89.2)$ & 4.9 & $9(24.3)$ & $25(67.5)$ & $23(62.2)$ & $6(16.2)$ & - \\
\hline
\end{tabular}

$M$ male, $F$ female, Ma macroscopic, Mi microscopic, $H B S A g$ hepatitis B surface antigen, anti-HCV hepatitis C virus antibody, ST solitary tumor, TS tumor size, $M T$ multiple tumor, VI vascular invasion, $P D$ poor differentiation, $M$ intrahepatic metastasis, TCA tumor capsule absence

${ }^{a}$ mean or median

${ }^{b}$ including 1 patients underwent liver transplantation; ${ }^{c}$ including 5 patients underwent liver transplantation; ${ }^{d}$ including 2 patients underwent liver transplantation 
Table 2 Operative intervention and outcomes

\begin{tabular}{|c|c|c|c|c|c|c|c|}
\hline References & $\mathrm{Hx} \pm \mathrm{Tb} n(\%)$ & $\mathrm{Hx}+\mathrm{BDR} n(\%)$ & Tb only n (\%) & Mortality n (\%) & $\begin{array}{l}\text { Median survival } \\
\text { (Months) }\end{array}$ & $\begin{array}{l}\text { Overall survival (\%) } \\
1 \text {-year 3-years 5-years }\end{array}$ & Recurrence $\mathrm{n}(\%)$ \\
\hline 5 & $12(70.6)$ & $5(29.4)$ & $0(0)$ & $0(0)$ & - & 583016 & $10 / 15(66.7)$ \\
\hline 6 & $12(70.6)$ & $5(29.4)$ & $0(0)$ & $0(0)$ & 17.6 & 754728 & $11(64.7)$ \\
\hline 7 & $7(50)$ & $4(28.5)$ & $3(21.4)$ & $1(7.1)$ & 14 & 735121 & $7(100)$ \\
\hline 8 & $30(88.2)$ & $1(2.9)$ & $1(2.9)$ & $1(2.9)$ & - & $7111-$ & $14 / 28(50)$ \\
\hline 9 & - & - & - & $1(5.9)$ & 20.8 & 60206.7 & $11 / 16(68.7)$ \\
\hline 12 & $33(86.8)$ & $5(13.2)$ & $0(0)$ & $0(0)$ & 31 & 794533 & $29(76.3)$ \\
\hline 13 & $12(80)$ & $3(20)$ & $0(0)$ & $0(0)$ & 11.4 & 46230 & $11(73.3)$ \\
\hline 14 & $40(93)$ & $3(7)$ & $0(0)$ & $1(2.1)$ & 37 & 935624 & $10(21)$ \\
\hline 15 & $20(90.9)$ & $2(9.1)$ & $0(0)$ & $0(0)$ & - & 623030 & $13 / 16(81.2)$ \\
\hline 16 & $26(96.3)$ & $1(3.7)$ & $0(0)$ & $1(3.7)$ & - & 70267.4 & $25 / 26(96.1)$ \\
\hline 17 & $12(66.7)$ & $6(33.3)$ & $0(0)$ & $2(10)$ & - & $7321-$ & $7 / 13(53.8)$ \\
\hline 18 & $42(57.5)$ & $31(42.5)$ & $0(0)$ & $3(4.1)$ & - & 774132 & $52 / 70(74.3)$ \\
\hline 19 & $25(71.4)$ & $10(28.6)$ & $0(0)$ & $0(0)$ & 19 & 382011 & - \\
\hline 20 & $7(53.8)$ & $6(46.2)$ & $0(0)$ & $0(0)$ & 47 & 927748 & $6(46.1)$ \\
\hline 21 & $37(84)$ & $7(16)$ & $0(0)$ & $2(4.5)$ & 23.7 & 703831 & $30 / 41(73.2)$ \\
\hline 22 & $31(100)$ & $0(0)$ & $0(0)$ & $0(0)$ & - & 9061 - & $20(64.5)$ \\
\hline 23 & $30(76.9)$ & $9(23.1)$ & $0(0)$ & $2(5.1)$ & 28.6 & 824810 & - \\
\hline 24 & $9(24.3)$ & $28(75.7)$ & $0(0)$ & $0(0)$ & 44 & 695439 & $23(62.1)$ \\
\hline 25 & 15 (78.9) & $4(21.1)$ & $0(0)$ & $0(0)$ & - & 823932 & $10 / 15(66.7)$ \\
\hline 26 & $23(62.2)$ & $14(37.8)$ & $0(0)$ & $0(0)$ & - & 642418 & $21(56.8)$ \\
\hline
\end{tabular}

$H x$ hepatectomy, $T b$ thrombectomy, BDR bile duct resection

Table 3 Results of meta-analysis comparing hepatocellular carcinoma with or without biliary tumor thrombus

\begin{tabular}{|c|c|c|c|c|c|c|}
\hline Outcome of interest & No. of studies & No. of patients BTT Non-BTT & OR/WMD & $95 \% \mathrm{Cl}$ & $P$ value & HG $P$ value \\
\hline \multicolumn{7}{|l|}{ Clinicopathological characteristics } \\
\hline Male & 12 & 3175806 & 1.07 & $0.97,1.43$ & 0.67 & 0.69 \\
\hline Age & 9 & 2563840 & 0.01 & $-1.01,1.02$ & 0.99 & 0.01 \\
\hline Hepatitis B surface antigen & 11 & 2975150 & 1.33 & $0.99,1.79$ & 0.06 & 0.35 \\
\hline Hepatitis C virus antibody & 9 & 2434274 & 0.72 & $0.37,1.39$ & 0.33 & 0.03 \\
\hline Cirrhosis & 6 & 1583569 & 0.72 & $0.51,1.00$ & 0.05 & 0.11 \\
\hline Tumor size & 7 & 1774028 & -0.89 & $-2.63,0.85$ & 0.32 & $<0.001$ \\
\hline Multiple tumor & 6 & 1663662 & 1.85 & $0.76,4.52$ & 0.18 & $<0.001$ \\
\hline Tumor capsule absence & 7 & 1762305 & 1.26 & $0.63,2.51$ & 0.62 & 0.02 \\
\hline Intrahepatic metastases & 5 & 1091710 & 1.47 & $0.93,2.33$ & 0.10 & 0.40 \\
\hline Vscular invasion & 12 & 2935939 & 4.70 & $2.90,7.60$ & $<0.001$ & 0.001 \\
\hline Poor differentiation & 8 & 2274003 & 2.07 & $1.23,3.49$ & 0.006 & 0.05 \\
\hline \multicolumn{7}{|l|}{ Long-term outcomes } \\
\hline 1-year overall survival & 12 & 2804739 & 0.48 & $0.31,0.72$ & $<0.001$ & 0.02 \\
\hline 3-year overall survival & 12 & 2804739 & 0.45 & $0.29,0.68$ & $<0.001$ & 0.007 \\
\hline 5-year overall survival & 10 & 2314021 & 0.31 & $0.21,0.64$ & $<0.001$ & 0.02 \\
\hline
\end{tabular}




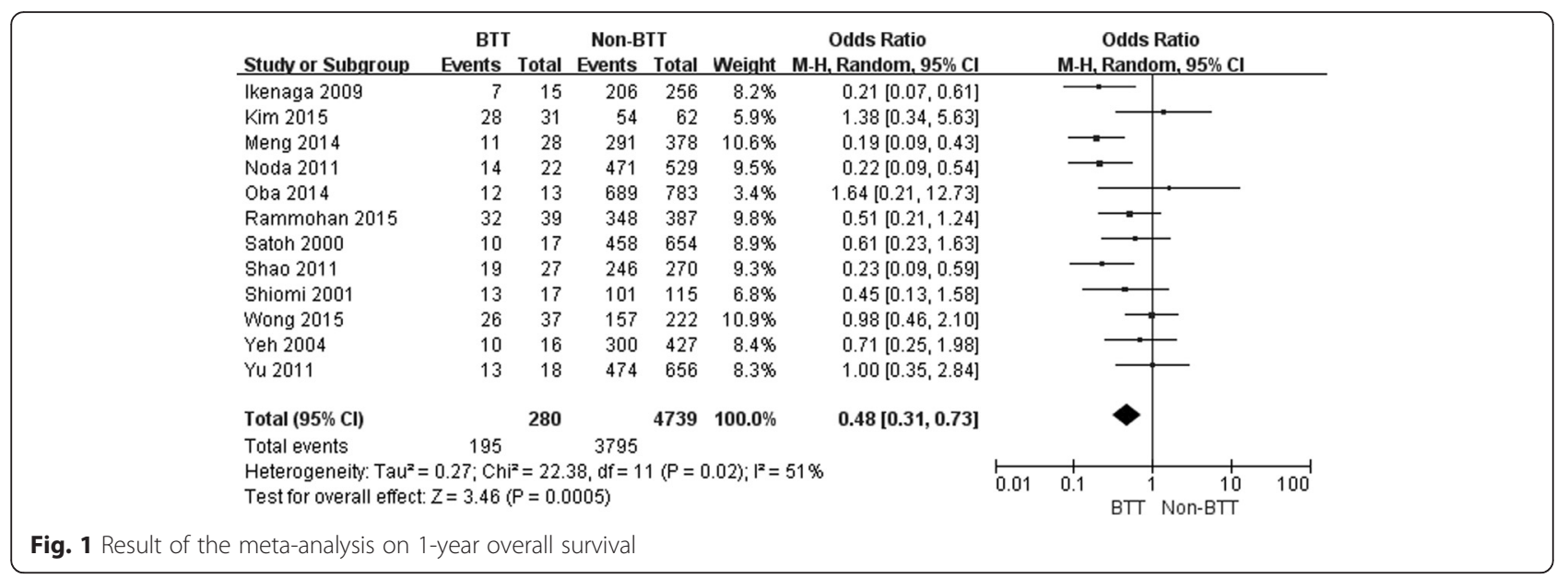

A funnel plot analyzing all studies utilized in the 1year OS analysis demonstrated asymmetry, suggesting the presence of publication bias (Fig. 4).

\section{Discussion}

HCC patients with BTT usually respond poorly to nonsurgical treatments, such as transcatheter arterial chemoembolization (TACE), percutaneous transhepatic biliary drainage, and radiation. Oba et al. [20] reported that the 1-, 3-, and 5-year OS rate in their 25 patients was $14 \%, 5 \%$ and $0 \%$ after nonsurgical treatment, respectively. Luo et al. [14] reported a 5-year OS rate of $0 \%$ for patients treated with TACE $(n=27)$ or biliary decompression $(n=40)$. In this study, patients who received surgical resection had a 5-year OS rate of $24 \%$, which is far better than the results of the nonsurgical treatments discussed above. So it seems justified to carry out surgical resection for this group of patients.

We found that long-term survival in patients with BTT was significantly shorter than that in patients without BTT. This may be a consequence of the fact that BTT has more aggressive biologically characteristics. As showed in the current study, the incidence of vascular invasion and poor differentiation, two powerful unfavorable prognostic factors, were more frequently observed in BTT group than those in non-BTT group.

The high incidence of postoperative recurrence limits the potential for surgical cure of HCC. Postoperative recurrence is usually classified as early ( $\leq 1$ year) and late ( $>1$ year) recurrence. Early recurrence was found to be associated with worse prognosis compared with late recurrence. ${ }^{27}$ Ikenaga et al. [13] reported that $53 \%$ of their patients in BTT group developed recurrences in the remnant liver within 3 months after surgery. Qin [8], Noda [15], Shao [16], Zeng [26] and their colleagues reported that more than $50 \%$ of their BTT patients experienced recurrences during the first year after surgery. Shao et al. [16] found that patients with BTT had a higher rate of early recurrence than those without BTT (70.3\% vs. $34.8 \% ; P<0.001$ ). In aggregate, these results suggest that patients with BTT had a higher propensity for early recurrence. It is widely accepted that early recurrence is mainly caused by intrahepatic metastasis from the primary tumor via the venous circulation [27].

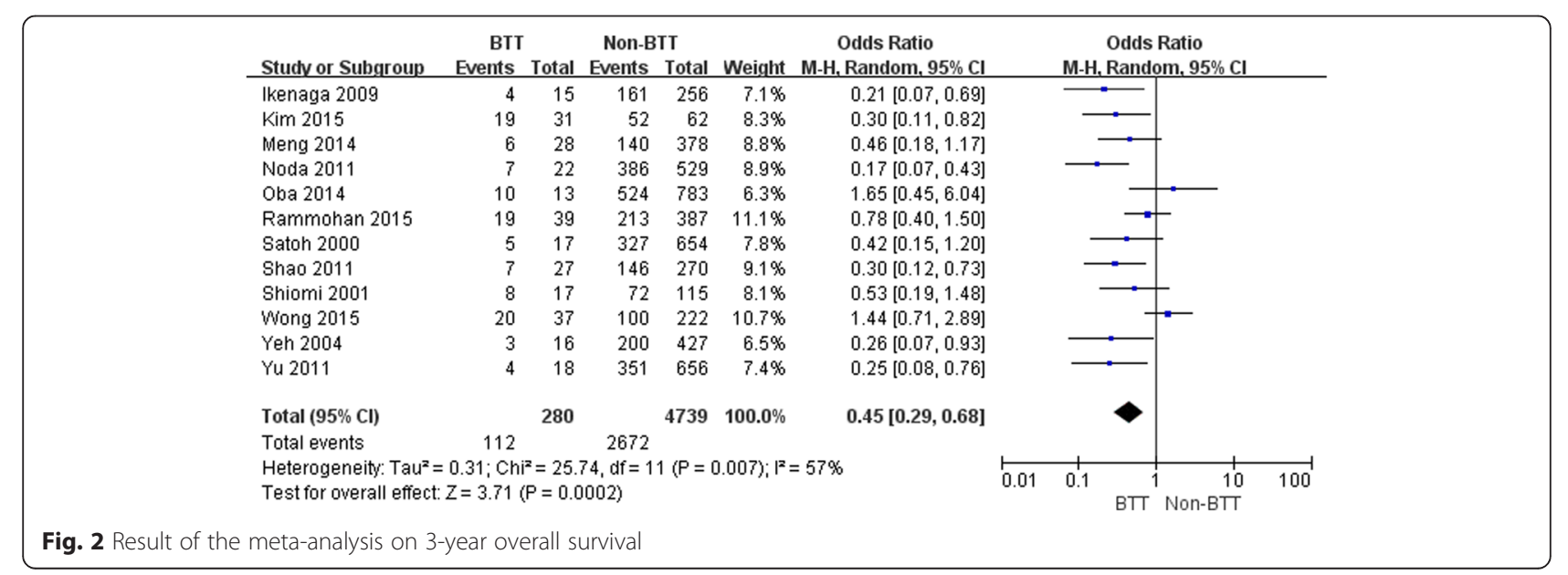




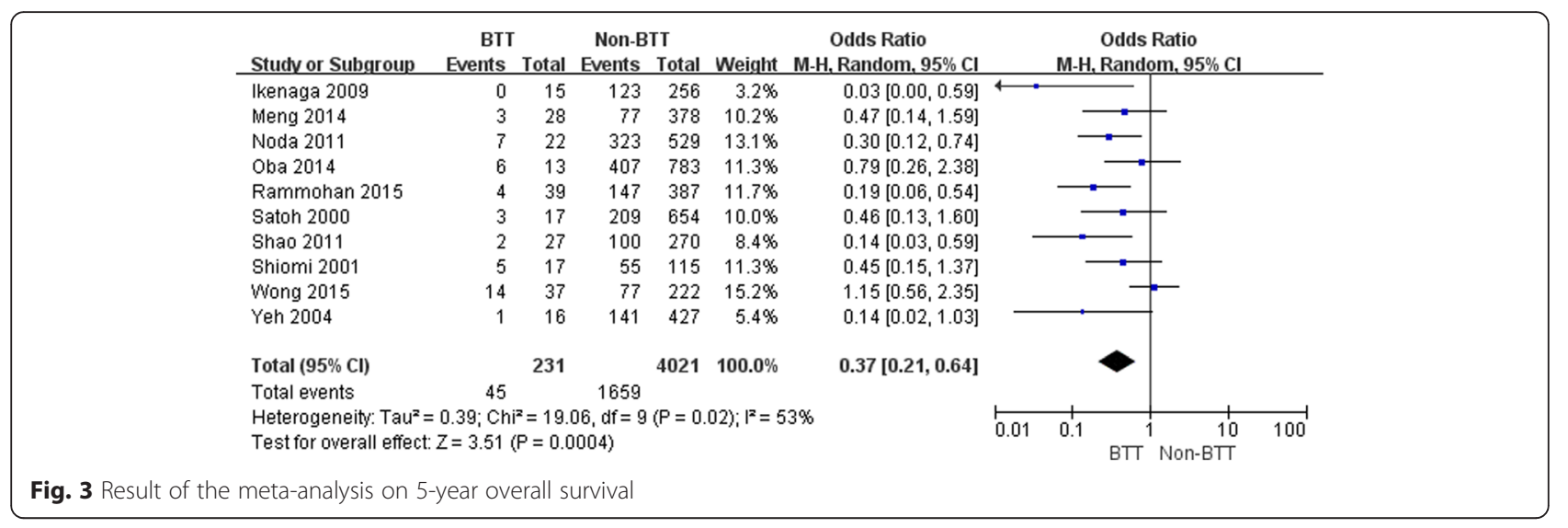

As most patients with BTT also had vascular invasion, it is understandable that BTT is likely to recur early after resection. On the other hand, Ikenaga et al. [13] reported two cases of BTT patients without vascular invasion who developed early recurrence in the remnant liver. Similarly, Shao et al. [16] reported nine cases of BTT patients without vascular invasion who experienced early recurrence. These data indicate that dissemination via the bile duct system is another gate of intrahepatic metastasis. In the American Joint Committee on Cancer (AJCC)/International Union Against Cancer (UICC) staging system, information of BTT is not required [28]. In contrast, in the Liver Cancer Study Group of Japan (LCSGJ) staging system, patients with BTT are assigned to the advanced stage and had a less favorable prognosis [29]. Based on the results of the present study, the LCSGJ staging system appears to be more appropriate for HCC lesions than the AJCC/UICC system.

The necessity of bile duct resection for $\mathrm{HCC}$ with macroscopic BTT is a subject of debate. Some authors found that BTT rarely invaded the bile duct wall around the hepatic hilus and could be easily removed $[5,6,15,25]$. Besides, analysis of the OS rate by some studies showed

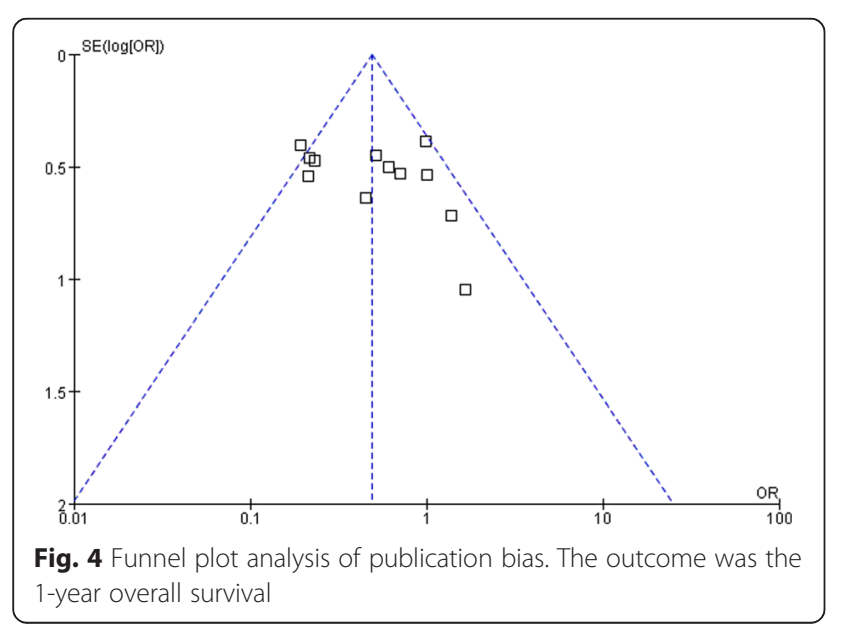

that bile duct resection did not seem to provide any significant benefit $[5,6,15]$, and therefore they suggested that such resection should be avoided unless essential for technical purposes. Whereas other authors advocated routine bile duct resection for $\mathrm{HCC}$ patients with BTT, knowing that it may minimize recurrence due to better eradication of the microscopic tumor [24, 26]. As all these studies involved the analysis of only a small number of patients, comparisons of the results between the two groups may be of limited value, and therefore future studies with a larger number of patients are required.

There have been relatively few studies in the literature reporting the practice and outcome of liver transplantation for the treatment of HCC with BTT. Peng et al. [7] reported one patient who died of recurrence at 27 months after liver transplantation. Hwang et al. [30] reported a 5year OS rate of $50 \%$ in a cohort of 14 patients. Although these authors believed that liver transplantation may be a potential treatment option for HCC with BTT, it is prudent to draw any firm conclusion before more results are obtained from further studies with larger sample sizes.

The present study has several limitations. First, all the included studies were performed in the Asia-Pacific region, which may affect the generalizability. In addition, the retrospective nature of eligible studies is vulnerable to introduce potential bias. For example, there were differences in observation periods, disease stages and surgical methods between the institutions. For this reason, significant heterogeneity was tested in the meta-analytic statistical outcomes. Finally, as only 13 of the 20 included studies were eligible for meta-analysis, the impact of BTT on the prognosis may be underestimated.

\section{Conclusions}

HCC with BTT has aggressive biological characteristics and is an indicator of poor prognosis. However, surgical resection can still provide long-term survival for some patients. More effective adjuvant therapies need to be developed to improve the outcome. Adoptive immunotherapy [31], 
antiviral therapy [32, 33], intrahepatic injection of 131Ilipiodol [34], and sorafenib- or peretinoin-based chemotherapy $[35,36]$ may provide beneficial effects.

\section{Competing interests}

The authors declare that they have no competing interests.

\section{Authors' contributions}

YZ and FY participated in the design and coordination of the study, carried out the critical appraisal of studies and wrote the manuscript. WQ, LW, and $\mathrm{BL}$ developed the literature search, carried out the extraction of data, assisted in the critical appraisal of included studies and assisted in writing up. LW,YZ, and FY carried out the statistical analysis of studies. All authors read and approved the final manuscript.

\section{Acknowledgements}

We thank Doctor Yanfang Zhao (Department of Health Statistics, Second Military Medical University, Shanghai, China) for her critical revision of the meta-analysis section.

\section{Author details}

'Department of General Surgery, First Hospital of Lanzhou University, Lanzhou, China. ${ }^{2}$ Department of Hepatobiliary Surgery, No.101 Hospital of CPLA, Wuxi, China. ${ }^{3}$ Department of Hepato-Biliary-Pancreato-Vascular Surgery, First affiliated Hospital of Xiamen University, Xiamen, China.

Received: 21 November 2015 Accepted: 25 January 2016

Published online: 28 January 2016

\section{References}

1. Llovet JM, Burroughs A, Bruix J. Hepatocellular carcinoma. Lancet. 2003; 362:1907-17.

2. Zhou YM, Yang JM, Li B, Yin ZF, Xu F, Wang B, et al. Risk factors for early recurrence of small hepatocellular carcinoma after curative resection. Hepatobiliary Pancreat Dis Int. 2010:9:33-7.

3. Kojiro M, Kawabata K, Kawano Y, Shirai F, Takemoto N, Nakashima T. Hepatocellular carcinoma presenting as intrabile duct tumor growth: a clinicopathologic study of 24 cases. Cancer. 1982;49:2144-7.

4. Huang JF, Wang LY, Lin ZY, Chen SC, Hsieh MY, Chuang WL, et al. Incidence and clinical outcome of icteric type hepatocellular carcinoma. J Gastroenterol Hepatol. 2002:17:190-5

5. Satoh S, Ikai I, Honda G, Okabe H, Takeyama O, Yamamoto Y, et al. Clinicopathologic evaluation of hepatocellular carcinoma with bile duct thrombi. Surgery. 2000:128:779-83.

6. Shiomi M, Kamiya J, Nagino M, Uesaka K, Sano T, Hayakawa N, et al. Hepatocellular carcinoma with biliary tumor thrombi: aggressive operative approach after appropriate preoperative management. Surgery. 2001;129:692-8.

7. Peng SY, Wang JW, Liu YB, Cai XJ, Xu B, Deng GL, et al. Hepatocellular carcinoma with bile duct thrombi: analysis of surgical treatment. Hepatogastroenterology. 2004:51:801-4.

8. Qin LX, Ma ZC, Wu ZQ, Fan J, Zhou XD, Sun HC, et al. Diagnosis and surgical treatments of hepatocellular carcinoma with tumor thrombosis in bile duct: experience of 34 patients. World J Gastroenterol. 2004;10:1397-401.

9. Yeh CN, Jan YY, Lee WC, Chen MF. Hepatic resection for hepatocellular carcinoma with obstructive jaundice due to biliary tumor thrombi. World J Surg. 2004;28:471-5.

10. Moher D, Liberati A, Tetzlaff J, Altman DG. Preferred reporting items for systematic reviews and meta-analyses: the PRISMA statement. BMJ. 2009; 339:b2535.

11. CEBM. Oxford Center for Evidence-Based Medicine:. http://www.cebm.net/ oxford-centre-evidence-based-medicine-levels-evidence-march-2009/. [accessed 30 July 2015].

12. Esaki M, Shimada K, Sano T, Sakamoto Y, Kosuge T, Ojima H. Surgical results for hepatocellular carcinoma with bile duct invasion: a clinicopathologic comparison between macroscopic and microscopic tumor thrombus. J Surg Oncol. 2005:90:226-32.

13. Ikenaga N, Chijiiwa K, Otani K, Ohuchida J, Uchiyama S, Kondo K. Clinicopathologic characteristics of hepatocellular carcinoma with bile duct invasion. J Gastrointest Surg. 2009;13:492-7.

14. Xiangij $L$, Weifeng T, Bin Y, Chen L, Xiaoging J, Baihe Z, et al. Surgery of hepatocellular carcinoma complicated with cancer thrombi in bile duct: efficacy for criteria for different therapy modalities. Langenbecks Arch Surg. 2009;394:1033-9.

15. Noda T, Nagano H, Tomimaru Y, Murakami M, Wada H, Kobayashi S, et al. Prognosis of hepatocellular carcinoma with biliary tumor thrombi after liver surgery. Surgery. 2011;149:371-7.

16. Shao W, Sui C, Liu Z, Yang J, Zhou Y. Surgical outcome of hepatocellular carcinoma patients with biliary tumor thrombi. World J Surg Oncol. 2011;9:2.

17. Yu XH, Xu LB, Liu C, Zhang R, Wang J. Clinicopathological characteristics of 20 cases of hepatocellular carcinoma with bile duct tumor thrombi. Dig Dis Sci. 2011;56:252-9.

18. Moon DB, Hwang S, Wang HJ, Yun SS, Kim KS, Lee YJ, et al. Surgical outcomes of hepatocellular carcinoma with bile duct tumor thrombus: a Korean multicenter study. World J Surg. 2013:37:443-51.

19. Meng KW, Dong M, Zhang WG, Huang QX. Clinical characteristics and surgical prognosis of hepatocellular carcinoma with bile duct invasion. Gastroenterol Res Pract. 2014;2014:604971.

20. Oba A, Takahashi S, Kato Y, Gotohda N, Kinoshita T, Shibasaki H, et al. Usefulness of resection for hepatocellular carcinoma with macroscopic bile duct tumor thrombus. Anticancer Res. 2014;34:4367-72.

21. Kasai Y, Hatano E, Seo S, Taura K, Yasuchika K, Uemoto S. Hepatocellular carcinoma with bile duct tumor thrombus: surgical outcomes and the prognostic impact of concomitant major vascular invasion. World J Surg. 2015:39:1485-93.

22. Kim JM, Kwon $\mathrm{CH}$, Joh JW, Sinn DH, Park JB, Lee JH, et al, Incidental microscopic bile duct tumor thrombi in hepatocellular carcinoma after curative hepatectomy: a matched study. Medicine (Baltimore). 2015:94:e450.

23. Rammohan A, Sathyanesan J, Rajendran K, Pitchaimuthu A, Perumal SK, Balaraman $\mathrm{K}$, et al. Bile duct thrombi in hepatocellular carcinoma: is aggressive surgery worthwhile? HPB (Oxford). 2015;17:508-13.

24. Wong TC, Cheung TT, Chok KS, Chan AC, Dai WC, Chan SC, et al. Outcomes of hepatectomy for hepatocellular carcinoma with bile duct tumour thrombus. HPB (Oxford). 2015;17:401-8.

25. Yamamoto S, Hasegawa K, Inoue Y, Shindoh J, Aoki T, Sakamoto Y, et al. Bile duct preserving surgery for hepatocellular carcinoma with bile duct tumor thrombus. Ann Surg. 2015;261:e123-5.

26. Zeng $H$, Xu LB, Wen JM, Zhang R, Zhu MS, Shi XD, et al. Hepatocellular carcinoma with bile duct tumor thrombus: a clinicopathological analysis of factors predictive of recurrence and outcome after surgery. Medicine (Baltimore). 2015:94:e364.

27. Poon RT, Fan ST, Ng IO, Lo CM, Liu CL, Wong J. Different risk factors and prognosis for early and late intrahepatic recurrence after resection of hepatocellular carcinoma. Cancer. 2000;89:500-7.

28. Chun YH, Kim SU, Park JY, do Kim Y, Han KH, Chon CY. Prognostic value of the 7th edition of the AJCC staging system as a clinical staging system in patients with hepatocellular carcinoma. Eur J Cancer. 2011;47:2568-75.

29. Minagawa M, Ikai I, Matsuyama Y, Yamaoka Y, Makuuchi M. Staging of hepatocellular carcinoma: assessment of the Japanese TNM and AJCC/UICC TNM systems in a cohort of 13,772 patients in Japan. Ann Surg. 2007:245:909-22.

30. Ha TY, Hwang S, Moon DB, Ahn CS, Kim KH, Song GW, et al. Long-term survival analysis of liver transplantation for hepatocellular carcinoma with bile duct tumor thrombus. Transplant Proc. 2014;46:774-7.

31. Lee JH, Lee JH, Lim YS, Yeon JE, Song TJ, Yu SJ, et al. Adjuvant immunotherapy with autologous cytokine-induced killer cells for hepatocellular carcinoma. Gastroenterology. 2015;148:1383-91.

32. Breitenstein $\mathrm{S}$, Dimitroulis $\mathrm{D}$, Petrowsky $\mathrm{H}$, Puhan MA, Müllhaupt $\mathrm{B}$, Clavien PA. Systematic review and meta-analysis of interferon after curative treatment of hepatocellular carcinoma in patients with viral hepatitis. $\mathrm{Br} J$ Surg. 2009;96:975-81.

33. Zhou Y, Zhang Z, Zhao Y, Wu L, Li B. Antiviral therapy decreases recurrence of hepatitis B virus-related hepatocellular carcinoma after curative resection: a meta-analysis. World J Surg. 2014:38:2395-402.

34. Lau WY, Lai EC, Leung TW, Yu SC. Adjuvant intra-arterial iodine-131-labeled lipiodol for resectable hepatocellular carcinoma: a prospective randomized trial-update on 5-year and 10-year survival. Ann Surg. 2008;247:43-8.

35. Wang SN, Chuang SC, Lee KT. Efficacy of sorafenib as adjuvant therapy to prevent early recurrence of hepatocellular carcinoma after curative surgery: A pilot study. Hepatol Res. 2014;44:523-31.

36. Okita K, Izumi N, Matsui O, Tanaka K, Kaneko S, Moriwaki H, et al. Peretinoin after curative therapy of hepatitis C-related hepatocellular carcinoma: a randomized double-blind placebo-controlled study. J Gastroenterol. 2015;50:191-202. 